\title{
Twenty Three
}

National Cancer Institute

\section{Source}

National Cancer Institute. Twenty Three. NCI Thesaurus. Code C126885.

A natural number greater than twenty-two and less than twenty-four and the quantity that it denotes. 